\title{
Mean QRS Duration
}

National Cancer Institute

\section{Source}

National Cancer Institute. Mean QRS Duration. NCI Thesaurus. Code C62087.

The average (mean) duration (time) of the QRS interval, obtained from a set of measurements of the QRS interval. The QRS interval is defined as the time from the beginning of the QRS complex to the end of the QRS complex, representing the time it takes for the ventricles to depolarize. 